EPJ Web of Conferences 41, 04016 (2013)

DOI: $10.1051 /$ epjconf/20134104016

(C) Owned by the authors, published by EDP Sciences, 2013

\title{
Ultrafast Surface-Plasmon Enhancement of Exciton and Defect Luminescence in ZnO Thin Films
}

\author{
B. J. Lawrie ${ }^{1}$, R. $\mathrm{Mu}^{2}$ and R. F. Haglund, Jr. ${ }^{3}$ \\ ${ }^{1}$ Oak Ridge National Laboratory, Oak Ridge, TN 37831-6418 USA \\ ${ }^{2}$ Department of Physics, Fisk University, Nashville, TN 37208 USA \\ ${ }^{3}$ Department of Physics and Astronomy, Vanderbilt University, Nashville, TN 37235-1807 USA
}

\begin{abstract}
Femtosecond pump-probe and photoluminescence measurements in transmission and reflection show that ultraviolet band-edge and visible defect luminescence in $\mathrm{ZnO}$ films can be selectively enhanced by coupling to $\mathrm{Ag}$ surface-plasmon polaritons or localized surface plasmon resonances.
\end{abstract}

\section{Introduction}

Photoluminescence spectra from zinc oxide ( $\mathrm{ZnO}$ ) thin films typically show an exciton recombination peak near $3.3 \mathrm{eV}$ and a broad, yellow-green PL band around $2.3 \mathrm{eV}$ due to donor-acceptor pair (DAP) recombination. The DAP PL band has been variously attributed to near band-edge holes with electrons localized at oxygen vacancies; near-conduction-band electrons with holes on oxygen vacancies or oxygen antisite defects. However, the identity of the multiple components of the donor-acceptor pair (DAP) visible luminescence remains to some extent a matter of conjecture.

While Purcell enhancement of band-edge PL and electroluminescence in $\mathrm{ZnO}$ has been reported in $\mathrm{ZnO}$ and in $\mathrm{Ag}-\mathrm{ZnO}$ heterostructures, we present here the first report of band-edge exciton dynamics in a defect-rich $\mathrm{ZnO}$ thin film where only defect dynamics were observed in the absence of plasmonic elements. The distinctive difference between band-edge and DAP dynamics has made it possible to identify one component of the broadband visible luminescence with the $\mathrm{Zn}_{i}$ interstitial defect. In addition, exciton-plasmon coupling makes it possible to increase band-edge luminescence by nearly two orders of magnitude where, in the absence of plasmonic coupling, only defect luminescence is observed. This suggests that plasmonic enhancement of exciton luminescence or specific defects - achievable by appropriate structuring of the plasmonic element — should be generally applicable to the problem of controlling defect recombination rates in opto-electronic devices.

\section{Experimental materials and methods}

Heterostructures comprising a $\mathrm{ZnO}$ film and a rough $\mathrm{Ag}$ film separated by an insulating $\mathrm{MgO}$ spacer layer were fabricated as follows [1]: ZnO films $50 \mathrm{~nm}$ thick were deposited on (0001) sapphire substrates by electron-beam evaporation (EBE), then annealed at temperatures between 400 and $800^{\circ} \mathrm{C}$ in a single-zone tube furnace under flowing oxygen $(9.8 \mathrm{ccm})$ or in

This is an Open Access article distributed under the terms of the Creative Commons Attribution License 2.0, which permits unrestricted use, distribution, and reproduction in any medium, provided the original work is properly cited. 
atmosphere. A $10 \mathrm{~nm}$ thick MgO film was deposited by EBE immediately after annealing to prevent creation of additional surface defect states. After masking half the $\mathrm{ZnO} / \mathrm{MgO}$ bilayer, a Ag film, nominally $30 \mathrm{~nm}$ thick, was evaporated onto the structure, as shown schematically in Figure 1(a). Degenerate pump-probe measurements with a pulse duration of approximately $150 \mathrm{fs}$ were then carried out using the fourth harmonic of an optical parametric amplifier at operated at $1430 \mathrm{~nm}$ [2]. Conventional photoluminescence spectroscopy was also employed to complement the pump-probe measurements. In both cases, the mask applied during Ag deposition made it possible to compare the optical response of identical $\mathrm{ZnO}-\mathrm{MgO}$ interfaces with and without plasmonic effects.

\section{Experimental results}

In the experimental configuration of Fig. 1(a), both the differential reflectivity and transmission for the heterostructure annealed at $400^{\circ} \mathrm{C}$ show a long-lived defectrecombination decay, but only the differential transmission in Fig. 1(b) shows the ps excitonrecombination response, indicating that the first several atomic layers near the $\mathrm{ZnO} / \mathrm{Al}_{2} \mathrm{O}_{3}$ interface support copious green-orange PL defect sites, but no band-edge recombination. The reflectivity exhibits the double-exponential decay profile known from the literature in which the longer-lived decay time is observed by two-color experiments on the orange and green PL dynamics [3].
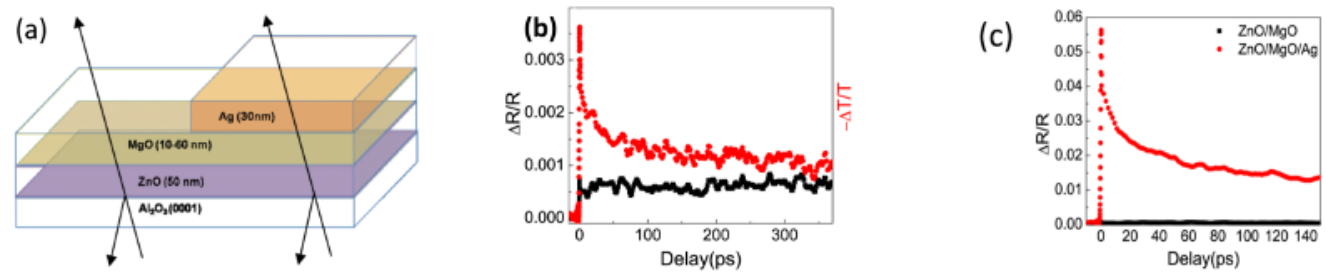

Fig. 1. (a) Experimental geometry showing substrate, $\mathrm{ZnO}$ film, MgO spacer layer, and rough Ag film. In Fig. 2, the sample was annealed in air at $400^{\circ} \mathrm{C}$. (b) Band-edge $(365 \mathrm{~nm})$ differential reflectivity and transmission as a function of pump-probe delay in the geometry of Fig. 1(a), for the heterostructure annealed at $400^{\circ} \mathrm{C}$. (c) Differential reflectivity for the sample of Fig. 1(b), with and without coupling to the plasmonic Ag film.

Figure 1(c) compares differential reflectivities for a sample with and without the Ag film. In contrast to Fig. 1(b), the differential reflectivity in this case shows a dramatic increase in the fast transient due to band-edge exciton decay when the Ag film is present, with a dominant 35 ps decay term that is absent on the uncoated sample. However, the presence of the Ag film allows for the emergence of near-interface band-edge recombination where none was observed in the heterostructure apart from the $\mathrm{Ag}$ film. Indeed, the peak reflectivity with the Ag film is 76 times as large as the peak reflectivity without the Ag film. This enhanced bandedge decay has previously been shown to be the result of exciton coupling to surface-plasmon polaritons in the Ag film [1].

The experimental configuration is reversed for the data of Fig. 2, with the pump and probe beams incident from the film side, as shown in Fig. 2(a). Figure 2(b) shows the differential reflection and transmission as a function of pump-probe delay for a sample annealed at $500^{\circ} \mathrm{C}$ in air, acquired in the geometry of Fig. 2(a) but on the portion of the sample without the Ag film. Moreover, the fact that this feature is seen in the differential reflectivity indicates that the defect sites responsible for the long-lived decay come primarily from very near the interface. The photoluminescence spectra show a strong enhancement of a luminescence feature at $2.91 \mathrm{eV}$ for the sample annealed in $\mathrm{O}_{2}$ that is not visible in the room temperature films [4], even 
with the silver film, as well as enhancement of the visible orange-green luminescence that results from coupling to localized surface plasmon resonances around $2 \mathrm{eV}$.
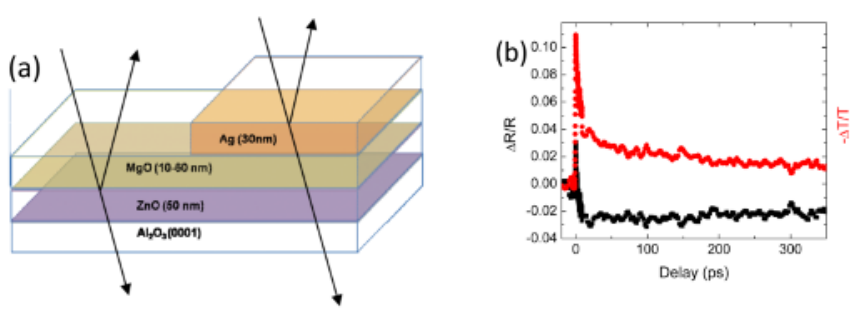

(c)

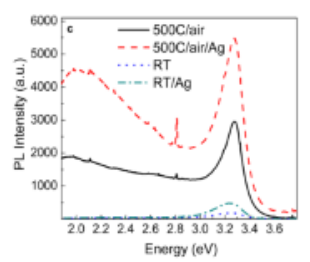

Fig. 2. (a) Experimental geometry showing substrate, $\mathrm{ZnO}$ film, MgO spacer layer, and rough Ag film. The sample was annealed in air at $500^{\circ} \mathrm{C}$. (b) Band-edge differential reflectivity and transmission as a function of pump-probe delay in the geometry of Fig. 1(a). The pump wavelength was $365 \mathrm{~nm}$. (c) Photoluminescence spectra for the same sample, showing the enhancement of the $\mathrm{Zn}_{i}$ defect $(2.91 \mathrm{eV})$ at the $\mathrm{ZnO}: \mathrm{MgO}$ interface for the sample with the Ag film, together with the band-edge and orangegreen defect luminescence.

\section{Discussion and conclusions}

We have shown in previous work that the $30 \mathrm{~nm}$ Ag film in our samples is topographically rough and can support both surface plasmon polaritons (SPPs) and localized surface plasmon resonances (LSPRs), the latter at gaps or asperities in the film. Calculations of the SPP dispersion curve for Ag films show that the plasmon density of states is maximal around $2.9 \mathrm{eV}$. Data presented in Fig. 1(c) show a Purcell enhancement of a feature at $2.91 \mathrm{eV}$ that we propose to identify with the $\mathrm{Zn}$ interstitial defect $\mathrm{Zn}_{i}$. X-ray photoelectron spectra of $\mathrm{ZnO}$ films annealed both in air and in oxygen atmosphere show that there is a relatively small population of $\mathrm{Zn}_{i}$ defects, so that its appearance in the annealed spectra in the presence of the $\mathrm{Ag}$ film can reasonably be ascribed to Purcell enhancement due to defect center-to-plasmon coupling.

It turns out that heterostructures of this type can be used generally to enhance the radiative decay rate of emission centers by coupling to localized or surface plasmons, thus potentially offering a new way of short-circuiting undesirable non-radiative decay channels in thin-film devices. This same technique has been applied, for example, to enhance the band-edge exciton intensity by more than two orders of magnitude compared to the green-orange defect emission in defect-rich samples produced by annealing in an extremely oxygen-rich atmosphere [5].

\section{Acknowledgements}

BJL and RFH acknowledge support for the ultrafast spectroscopy at Vanderbilt from the US Department of Energy, Office of Science (DE-FG02-01ER45916). PL and thin-film fabrication at Fisk University were supported by an NSF-CREST grant (HRD-0420516) and by a Department of Defense grant (W911NF-11-1-0156). The Oak Ridge National Laboratory is managed by UTBattelle, LLC, under Contract No. DE-AC05-00OR22725 with the U.S. Department of Energy.

\section{References}

1. B. J. Lawrie, R. Mu and R. F. Haglund, Jr., Opt. Express 17, 4637 (2009).

2. R. F. Haglund, Jr., B. J. Lawrie and R. Mu, Thin Solid Films 458, 4637 (2010).

3. C. Bauer, G. Boschloo, E. Mukhtar and A. Hagfeldt, Chem. Phys. Lett. 387, 176 (2004).

4. B. J. Lawrie, R. Mu and R. F. Haglund, Jr., Opt. Lett. 37, (2012).

5. B. J. Lawrie, R. Mu and R. F. Haglund, Jr., Plasmonics, submitted (2012). 